\title{
Le centre des mouvements de l'eau (CME), simulateur du comportement hydraulique et qualité du réseau du Syndicat des Eaux d'Ile de France
}

\author{
Linking SCADA and hydraulic and water quality simulator at the Centre \\ des Mouvements de l'Eau (CME) in Paris (France)
}

par V. Tiburce, J. -L. Gagnon, J. - L. Hamon

Générale des Eaux

P. Chopard

Syndicat des Eaux d'Ile-de-France

Ph. Feugier

PROLOG Ingénierie

The Centre des Mouvements de l'Eau (CME), operational since 1991 is a centralized Supervisory Control And Data Acquisition (SCADA) system for the water production and distribution network of the Syndicat des Eaux d'lle de France (SEDIF) which serves approximately 4,000,000 inhabitants around Paris (France). In 1994, the SEDIF and its operator, Générale des Eaux (VIVENDI group) initiated a project called the 'CME-phase II' project with the aim of linking the CME SCADA system with a network analysis software (the Stoner Workstation Service) capable of simulating the hydraulic and water quality operation of the water network. This application is operational since 1997 and provides the SEDIF with a new set of tools to better supervise the operation of the network, predict potential problems in the network and validate different operational strategies. On-line simulations (i.e. in parallel to the real system operation) allows for anomaly detection and resolution : a new set of operational data is received from the SCADA system at a predefined time interval and is used to update the hydraulic model before executing a steady state simulation. Key points in the network can be closely controlled (without supplementary monitoring equipment) and potential discrepancies between calculated and observed data can be detected and analyzed. The entire process is fully automatic and does not require any operator intervention. Off-line past simulations allow the operator to replay an event that took place in the network using the operational data archived by the SCADA system with the objective of better understanding the hydrailic and water quality behavior of the network. Off-line predictive simulations allow the operator to test various operational strategies. Using as a starting point the newest set of operational data and defining the network demand and the facilities operation in the coming hours or days, the operator can initiate $a$ variable simulation and assess the consequences of a specific operational strategy. The CME phase II application is also extensively used to validate any extension or reinforcement project as it permits a quick extraction and validation of SCADA data to calibrate a hydraulic model before assessing the impact of a new extension or reinforcement scheme.

\section{I - PRÉSENTATION DU RÉSEAU DU SYNDI- CAT DES EAUX D'ILE DE FRANCE}

Le Syndicat des Eaux d'lle de France (SEDIF) regroupe 144 communes de la banlieue parisienne et alimente en eau potable plus de 4 millions d'habitants, représentant près de 500000 clients domestiques et industriels.

Les ressources en eau du Syndicat proviennent dans leur quasi-totalité des 3 grands cours d'eau de la région parisienne :

- I'usine de Choisy-le-Roi traite l'eau de la Seine (capacité de production de $800000 \mathrm{~m}^{3 /} / \mathrm{jour}$ ) et alimente le secteur Sud du réseau de distribution,
- l'usine de Méry-sur-Oise traite l'eau de l'Oise (capacité de production de $270000 \mathrm{~m}^{3} /$ jour) et alimente le secteur Nord du réseau de distribution,

- I'usine de Neuilly-sur-Marne traite l'eau de la Marne (capacité de production de $800000 \mathrm{~m}^{3} / \mathrm{jour}$ ) et alimente le secteur Est du réseau de distribution.

Le réseau de distribution est constitué de $8500 \mathrm{~km}$ de conduites, de 45 usines de surpression, et de 51 réservoirs représentant une capacité de stockage d'environ $700000 \mathrm{~m}^{3}$.

La conduite des ouvrages du Syndicat est décentralisée et répartie au niveau des 3 usines de production, qui disposent chacune d'un poste de conduite permettant de piloter :

- l'usine de production elle-même, avec sa filière de traite- 
ment et les pompages élévatoires alimentant le réseau,

- l'ensemble des ouvrages du réseau de distribution sous sa dépendance : réservoirs, usines de surpression, vannes d'intercommunication, etc.

\section{II — LE SYSTÈME “ CME PHASE 1 ” DE SUPERVISION CENTRALISÉE}

Mis en place en 1991, le système de supervision centralisée CME est relié en temps réel aux systèmes de contrôlecommande des 3 usines de production, afin de permettre l'acquisition, l'archivage, et l'exploitation de l'ensemble des données de fonctionnement du réseau.

Le système CME s'appuie par ailleurs sur une application Minitel permettant aux exploitants du réseau de distribution de renseigner et d'horodater " manuellement " toutes les actions d'ouverture / fermeture effectuées sur les vannes qui ne sont raccordées à aucun système de contrôle-commande : le système CME supervise ainsi en " quasi temps réel " la position d'environ 5000 vannes stratégiques du réseau de distribution.

A partir de l'ensemble des données acquises, le système CME calcule en temps réel et archive un ensemble de grandeurs complémentaires, dont notamment la demande instantanée des 40 zones de distribution de l'exploitation.

Le système CME dispose également d'un module de prévision de la demande pour chacune des 40 zones de distribution.

\section{III — PRÉSENTATION DU PROJET "CME PHASE 2"}

L'objectif du projet CME Phase 2 était de coupler au système de supervision un simulateur du comportement hydraulique et qualité du réseau du SEDIF.

De manière générale, un tel outil permet :

- de calculer et de surveiller des paramètres hydrauliques (pression, débits) et qualité (résiduel de chlore, zones d'influence des différentes sources de production) au cœur du réseau de distribution, ce que ne permet pas le système de supervision CME,

- de simuler et de prévoir le comportement dynamique du réseau en fonction de différentes stratégies d'exploitation des ouvrages.

Le nouveau système CME Phase 2 a ainsi été construit autour des 4 grands objectifs fonctionnels suivants :

- simulation "temps réel" :

L'objectif est ici d'effectuer de manière automatique et permanente, à intervalles réguliers, un calcul statique sur l'ensemble du réseau, afin de :

- surveiller les principales grandeurs hydrauliques du réseau,

- détecter d'éventuels écarts entre valeurs calculées et valeurs acquises qui pourraient révéler de possibles dysfonctionnements au sein du réseau (dérive d'un capteur, information erronée sur la position d'une vanne, etc.)

- simulation "temps différé" :

Cette fonction permet à l'opérateur de "redérouler" un scénario passé, sur la base des données archivées par le système de supervision CME.

L'objectif est ici, typiquement, d'analyser le comportement hydraulique et qualité du réseau au cours de la journée écou- lée, et de pouvoir éventuellement détecter des anomalies de fonctionnement.

- simulation "prévisionnelle" :

Cette simulation permet de tester à l'avance différentes stratégies d'exploitation des usines et du réseau, et d'analyser leurs conséquences sur le comportement hydrodynamique du réseau.

En utilisant le module de prévision de la consommation implanté dans le système de supervision CME, cette simulation permet également de prévoir le comportement du réseau dans les prochaines heures, en partant des dernières conditions acquises par le superviseur.

- simulation " études/projets" :

L'application CME Phase 2 est également un instrument d'études et de validation de projets. Le SEDIF sera par exemple en mesure de définir les caractéristiques et d'analyser l'impact hydraulique de futures conduites, ou de valider les points d'implantation de nouveaux ouvrages de régulation ou de production, en confrontant à chaque fois le projet à des scénarios d'exploitation réels archivés par le système CME.

\section{IV — PRINCIPES DE FONCTIONNEMENT ET ARCHITECTURE DU SYSTEME "CME PHASE 2"}

Le système " CME Phase 2 " est bâti autour des éléments structurants suivants :

- utilisation du logiciel de simulation SWS, de la société américaine Stoner Associates,

- modélisation du réseau du SEDIF à travers une bibliothèque de modèles de références, préalablement construits et calés avec le logiciel SWS,

- construction autour du progiciel Oracle d'une base de données spécifique pour l'archivage des données superviseur, dédiée aux calculs de simulation,

- développement d'un outil de génération automatique des fichiers de simulation, s'appuyant sur les fonctionnalités du logiciel SWS pour la prise en compte de données superviseur,

- possibilité de transférer les résultats de simulations au sein d'un système d'information géographique,

- développement d'une interface opérateur globale, permettant à l'utilisateur de piloter facilement les différentes étapes de création, d'exécution, puis d'exploitation des résultats d'une simulation.

\section{- 4.1 Modélisation du réseau du SEDIF}

Déjà utilisé par le Syndicat pour ses travaux d'étude, le logiciel de simulation SWS, de la société américaine Stoner Associates, présente notamment les avantages suivants :

- capacité à gérer des réseaux de grande taille,

- fonctionnalités très complètes pour la prise en compte d'asservissements complexes sur les ouvrages, ainsi que pour la modélisation de la qualité de l'eau,

- capacités d'interface avec des superviseurs, incluant un module spécifique de simulation temps réel.

Les 40 zones de distribution du réseau du Syndicat ont été regroupées au sein de 10 modèles unitaires correspondant chacun aux 10 sous-réseaux piézométriques principaux du réseau. 
Chacun de ces modèles est relativement détaillé puisqu'en fonction de la nature de la zone modélisée (réseau de transport de lère élévation, ou réseau fin de 2ème ou 3ème élévation), les conduites prises en compte sont en général d'un diamètre supérieur à 150 ou $200 \mathrm{~mm}$, ce qui représente environ $25 \%$ du linéaire total du réseau.

Chaque modèle a par ailleurs été construit selon 2 " versions " distinctes :

- les modèles utilisés pour la simulation " prévisionnelle" intègrent une description complète des stations de pompage, avec les différents groupes et leur règles d'asservissement,

- les modèles utilisés pour la simulation " temps réel " ou " temps différé " reposent sur une description simplifiée des stations de pompage, et prennent en compte uniquement les données globales transmises par le superviseur (typiquement : le débit global de la station) afin de rejouer exactement le scénario passé.

\subsection{Archivage des données en provenance du superviseur}

Pour l'archivage des données superviseur, une base de données spécifique a été mise en place, intégrant uniquement les données nécessaires à la simulation hydraulique et qualité, et reposant sur :

- une sauvegarde au pas de temps de $10 \mathrm{mn}$ des valeurs analogiques (environ 1000 variables),

- un archivage de tous les changements d'état des grandeurs tout ou rien (environ 3000 variables),

- un archivage en ligne des 14 derniers mois.

Les variables analogiques prises en compte concernent environ :

- 400 valeurs de débits (débits groupe, débits station, débits de détente ou d'intercommunication,...),

- 200 mesures de pressions,

- 100 niveaux de cuve,

- 200 consignes ou mesures de résiduel de chlore,

- 80 valeurs de demande réseau (valeurs calculées ou valeurs prévisionnelles).

Les variables tout ou rien prises en compte concernent environ :

- 900 positions de vanne en ouvrage,

- 1800 états de bief du réseau de distribution,

- 150 états marche/arrêt de groupe.

\section{- 4.3 Principe de génération des données de simulation}

L'objectif recherché au cours du projet était de fournir aux futurs utilisateurs de l'application un environnement garantissant au maximum la cohérence et la " calculabilité " des données d'entrée de la simulation, quel que soit le scénario modélisé.

La démarche retenue, résumée par la figure 1 , a été la suivante :

- pour exécuter une simulation, l'opérateur CME Phase 2 utilise exclusivement des modèles préalablement calés et testés par des ingénieurs hydrauliciens, ces modèles étant sau- vegardés dans une " bibliothèque " de modèles,

- ces modèles contiennent des données " statiques " (description des nœuds et des tronçons composant le modèle, caractéristiques des ouvrages, répartition spatiale de la consommation, etc.) qui ne seront pas modifiées lors de la simulation, et un jeu de données dynamiques de référence (niveau des réservoirs, demande des réseaux, débit des ouvrages, état marche/arrêt des équipements, ouverture / fermeture des vannes, etc.) qui seront modifiées par le scénario de la simulation,

- lors de la création d'une simulation, le système CME Phase 2 génère un nouveau fichier de données dynamiques et le transmet au simulateur SWS avec une copie du fichier modèle de la bibliothèque,

- SWS met à jour uniquement les nouvelles données dynamiques de la simulation, garantissant ainsi au maximum la cohérence du modèle utilisé pour la simulation.

\subsection{Organisation générale de l'interface opérateur}

Le système CME Phase 2 offre une interface opérateur permettant de piloter de manière centralisée et simplifiée les différentes étapes d'exécution d'une simulation (figure 2) :

- pour définir les données d'entrée de la simulation qu'il

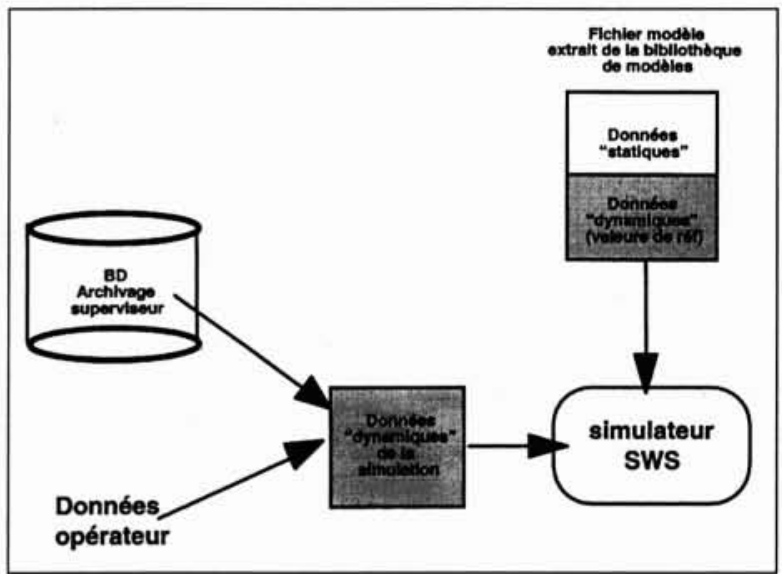

1. Principe de génération des données de simulation

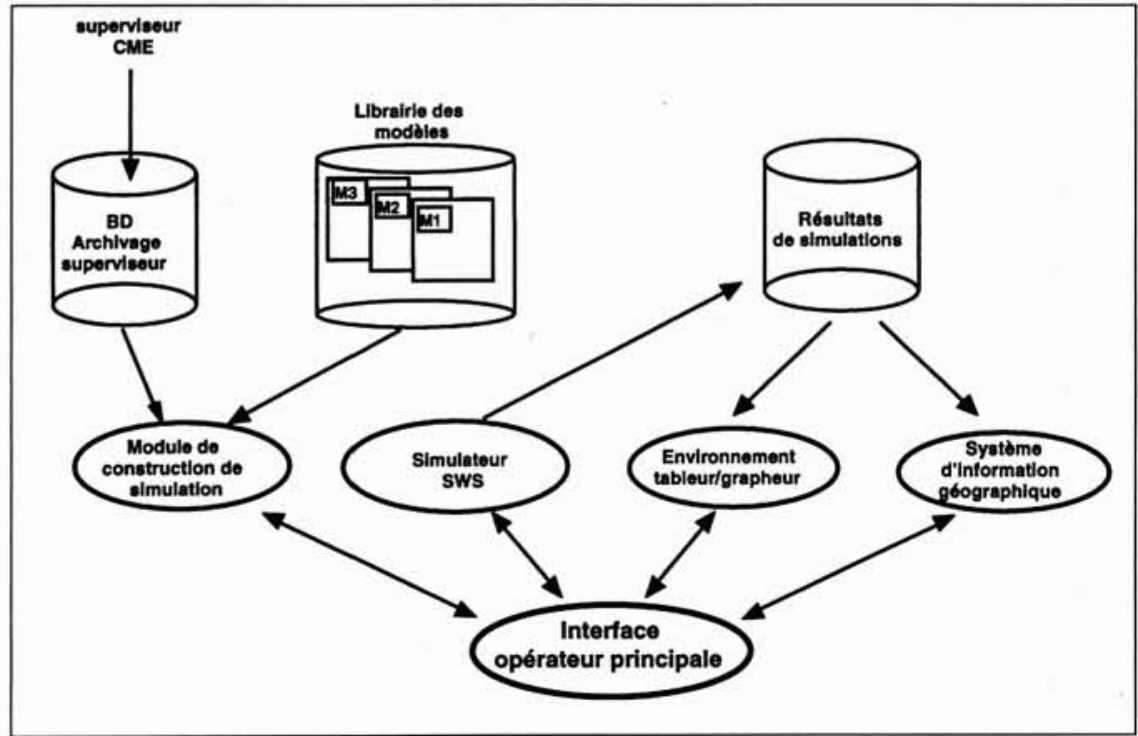

2. Organisation simplifiée du système CME Phase 2 


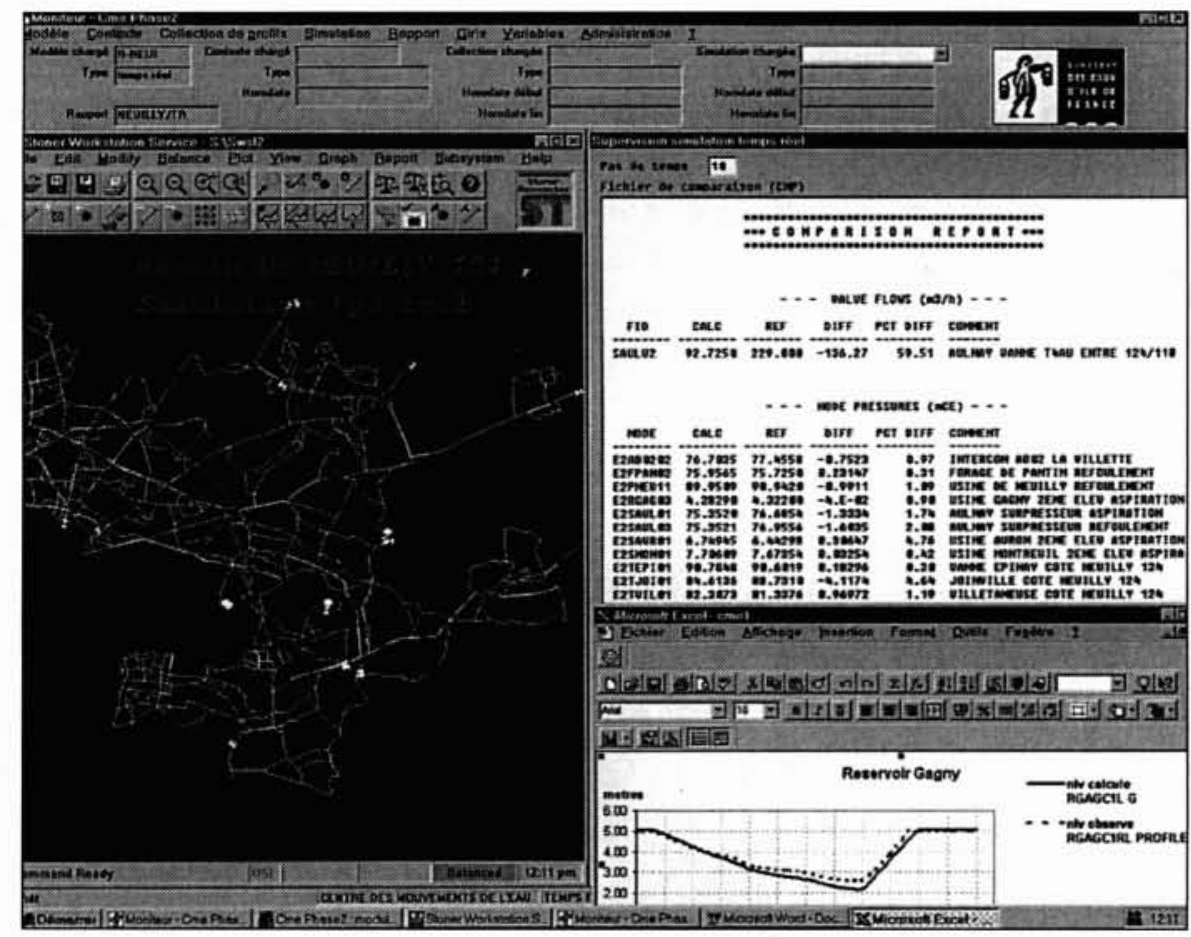

3. Vue écran CME Phase 2 pour le suivi des résultats temps réel souhaite exécuter (type de simulation, sélection du modèle, période temporelle étudiée,...), l'opérateur accède à un module de construction de simulation, qui permet de générer puis de transmettre automatiquement au logiciel SWS les fichiers de données nécessaires,

- l'opérateur exécute ensuite la simulation sous SWS, et peut s'il le souhaite exploiter et analyser les résultats de la simulation directement sous SWS,

- l'opérateur peut également exploiter et comparer les résultats de différentes simulations sous un environnement tableur/grapheur de type Excel,

- l'opérateur peut enfin transférer puis exploiter les résultats de la simulation au sein d'un système d'information géographique (affichage des résultats de la simulation sur fonds de plan cartographiques, requêtes et affichages thématiques spécifiques, etc.).

\section{V — LA SIMULATION " TEMPS RÉEL "}

La simulation temps réel exécute de manière automatique, toutes les 10 minutes, un calcul statique sur l'ensemble du réseau du Syndicat.

Cette fonction s'appuie étroitement sur le module temps réel " OLM" (On Line Module) du logiciel SWS.

Les résultats de ces calculs temps réel sont conservés sur une période glissante de 24 heures.

L'opérateur CME peut analyser les résultats de la simulation temps réel de plusieurs manières :

- un fichier de comparaison entre données calculées par SWS et données acquises par le superviseur est affiché en permanence, permettant de surveiller d'éventuels écarts qui pourraient révéler soit un problème capteur, soit un dysfonctionnement sur le réseau,

- l'opérateur peut visualiser les résultats des derniers calculs directement sous SWS,

- l'opérateur peut également visualiser les derniers calculs temps réel à travers différentes courbes de tendance Excel.
La figure 3 présente un exemple d'une vue écran CME Phase 2 pour le suivi des résultats temps réel.

\section{VI — LA SIMULATION “ TEMPS DIFFÉRÉ ”}

Cette simulation permet à l'opérateur de " redérouler " un scénario hydraulique et qualité passé, à l'aide des données acquises par le superviseur.

Après sélection d'un modèle de la bibliothèque de modèle, l'opérateur indique uniquement la fenêtre temporelle passée qu'il souhaite étudier, et le module de construction de simulation génère automatiquement les fichiers d'entrée de la simulation.

L'opérateur exécute ensuite la simulation sous SWS et peut exploiter les résultats soit directement sous SWS, soit à travers l'environnement tableur/grapheur Excel.

La figure 4 montre un exemple de résultat d'une simulation " temps différé " exécutée sur le réseau de Villejuif, où l'opérateur peut visualiser et comparer :

- les courbes des niveaux calculé et observé du réservoir de Villejuif,

- les courbes des valeurs calculées et observées du résiduel de chlore au sein du même réservoir de Villejuif.

Lorsque l'opérateur exécute une simulation " temps différé " incluant le calcul de paramètres qualité, il est amené à sélectionner une période temporelle d'environ 72 heures minimum, afin de permettre au simulateur SWS de simuler correctement la diffusion du chlore au sein du réseau ; les résultats " qualité " ne sont alors exploitables que sur la deuxième partie de la simulation (les 24 dernières heures par exemple).

\section{VII — LA SIMULATION “ PRÉVISIONNELLE ”}

Cette simulation permet de prévoir le comportement futur du réseau, en testant différents scénarios d'exploitation.

En fonction du scénario qu'il souhaite tester, l'opérateur peut sélectionner au sein de la bibliothèque un modèle " unitaire " décrivant un seul réseau piézométrique, ou bien choi- 


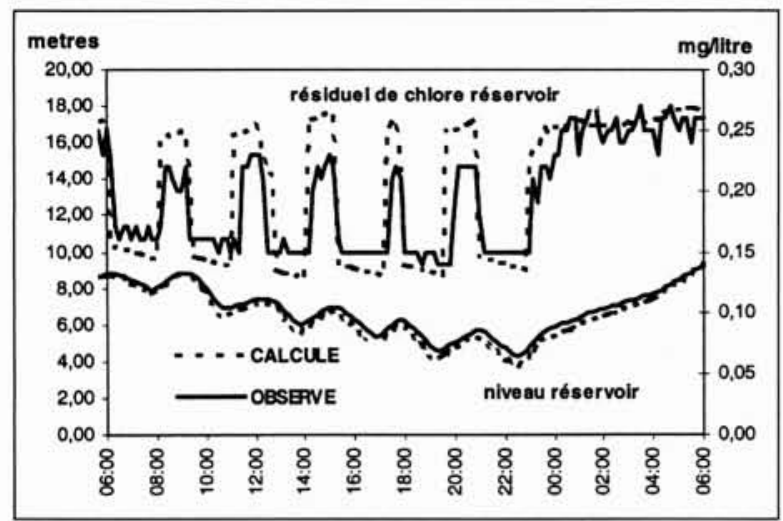

4. Simulation temps différé.

Calcul hydraulique et qualité sur $72 \mathrm{~h} /$ affichage des 24 dernières heures

sir un modèle plus global intégrant tout ou partie des différents réseaux de l'exploitation.

\section{ler exemple d'utilisation :}

L'opérateur souhaite, à $18 \mathrm{~h}$, prévoir le comportement du réseau de Montfermeil durant la nuit jusqu'à $6 \mathrm{~h}$ du matin. II exécute pour cela une simulation prévisionnelle qui repose sur :

- la prise en compte des conditions hydrauliques à $18 \mathrm{~h}$ (niveau du réservoir, pompes en fonctionnement au sein de l'usine de pompage),

- la prévision de demande,

- les règles d'asservissement de l'usine de pompage.

La figure 5 montre une comparaison " a posteriori " entre la simulation prévisionnelle et les valeurs effectivement observées par le superviseur.

\section{2ème exemple d'utilisation :}

L'opérateur veut tester les conséquences hydrauliques de l'isolement d'un bief important $(800 \mathrm{~mm})$ non loin de la sortie de l'usine de pompage de Gagny, entre $9 \mathrm{~h}$ du matin et $17 \mathrm{~h}$.

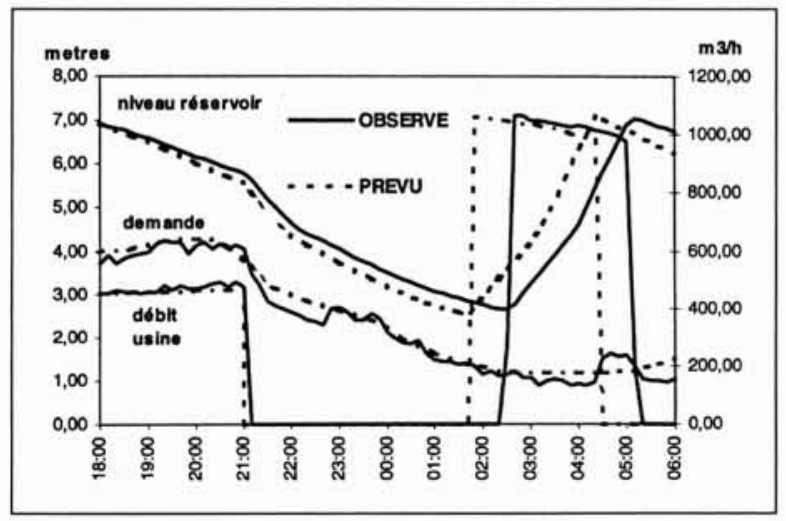

5. Simulation prévisionnelle - comparaison " a posteriori " entre grandeurs prévues et observées

A l'aide de l'outil CME Phase 2, il peut facilement simuler cette opération, et comparer la valeur du niveau piézométrique atteint en sortie d'usine avec celui correspondant à une situation normale (figure 6).

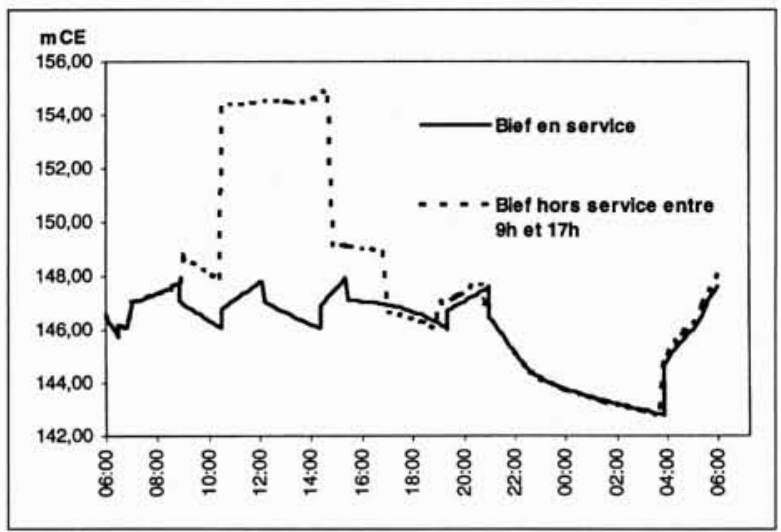

6. Simulation prévisionnelle - mise hors service d'un bief Conséquence sur le niveau piézométrique en sortie d'usine

\section{3ème exemple d'utilisation :}

La consigne de chloration en sortie de l'usine des Sorbiers, qui alimente le réservoir de Villejuif, est de $0,4 \mathrm{mg} /$ litre.

L'opérateur souhaite évaluer les conséquences d'un passage de la consigne à $0,3 \mathrm{mg} /$ litre en différents points tests du réseau, notamment au niveau du réservoir de Villejuif.

A l'aide de l'outil CME Phase 2, l'opérateur peut exécuter 2 simulations correspondant à chacune des 2 consignes, et comparer graphiquement les résultats (figure 7).

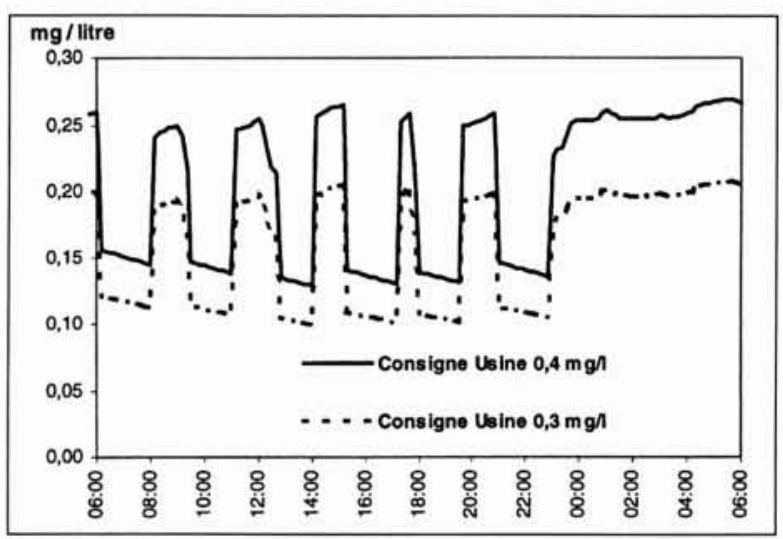

7. Simulation prévisionnelle - abaissement d'une consigne de chloration en sortie d'usine.

Conséquence sur le résiduel de chlore au sein du réservoir de Villejuif.

\section{VIII — CONCLUSIONS}

A travers le système " CME Phase 2 ", le Syndicat des Eaux d'Ile-de-France s'est doté d'un nouvel outil de surveillance et d'aide à I'exploitation de son réseau, qui vient compléter et renforcer l'architecture actuelle des systèmes de contrôle-commande et de supervision.

L'application CME Phase 2 fournit également désormais au Syndicat un instrument puissant d'études et de validation de projets.

Si la " prise en main " du système et l'analyse des retours d'expérience prendront probablement plusieurs mois, le nouveau système a d'ores et déjà permis d'analyser et de mieux comprendre certains comportements hydrauliques et qualité du réseau ; il a également permis de mettre en évidence et de corriger certaines dérives capteurs. 